\title{
Long-Term Use of Anti-Coronary Heart Disease Medications May Impact the Serum Zinc Concentration
}

\author{
Jiayun $\mathrm{Shi}^{1}{ }^{1} \cdot$. Lianping $\mathrm{He}^{1}$ (])
}

Received: 21 December 2021 / Accepted: 9 January 2022 / Published online: 12 January 2022

(c) The Author(s), under exclusive licence to Springer Science+Business Media, LLC, part of Springer Nature 2022

Dear editor,

We were pleased to read the research article by Meng et al. [1], who found that a decreased zinc ion concentration in the peripheral blood could be used as an independent risk factor for the prediction of coronary heart disease, especially in older patients, non-smokers, and women (especially postmenopausal women). Although this finding may have an important role in the prevention and treatment of coronary heart disease, some questions warrant further discussion.

Specifically, the research results are not consistent with a previous study that focused on plasma zinc as a marker for coronary artery disease and showed trace metals have dubious value as markers for coronary atherosclerosis [2]. Another study reported that the ratio of zinc-to-copper may be a preponderant factor in the etiology of coronary heart disease [3].

The basis for the relationship between the zinc ion concentration and coronary heart disease in the Meng et al. study [1] should be confirmed. First, the coronary heart disease group differed from the control group with respect to age, smoking history, co-existing diabetes or hypertension, and blood glucose, total cholesterol, high-density lipoprotein, low-density lipoprotein, and zinc levels. Furthermore, anti-coronary heart disease medications also may affect the level of serum zinc ions. Meng et al. [1] did not consider the effects of taking medications on serum zinc concentrations.
Indeed, we were unable to determine whether coronary heart disease and healthy controls were taking medications prior to blood collection. Overall, medication use was not an exclusion criterion for coronary heart disease patients or healthy controls. The long-term use of an anti-coronary heart disease medication may impact the serum zinc concentration.

\section{Declarations}

Conflict of Interest The authors declare no competing interests.

\section{References}

1. Meng HY, Wang YY, Zhou FF, Ruan JJ, Duan MY, Wang X et al (2021) Reduced serum zinc ion concentration is associated with coronary heart disease. Biol Trace Elem Res 199:4109-4118

2. Tiber AM, Sakhaii M, Joffe CD, Ratnaparkhi MV (1986) Relative value of plasma copper, zinc, lipids and lipoproteins as markers for coronary artery disease. Atherosclerosis 62:105-110

3. Klevay LM (1975) Coronary heart disease: the zinc/copper hypothesis. Am J Clin Nutr 28:764-774

Publisher's Note Springer Nature remains neutral with regard to jurisdictional claims in published maps and institutional affiliations.
Lianping $\mathrm{He}$

lianpinghe@tzc.edu.cn

1 School of Medicine, Taizhou University, Taizhou 318000,

Zhejiang, China 